\title{
Therapeutic Potential of Palm Wine on Selected Diarrhoeagenic
} \section{Bacteria}

\author{
E.J. Olotu*, O. Oluyele, O. R. Ojo, I. Osinowo and E. T. Ajimoko \\ Department of Microbiology, Faculty of Science, Adekunle Ajasin University, Akungba Akoko, Ondo State, \\ Nigeria
}

*Corresponding Author: E.J. Olotu, Department of Microbiology, Faculty of Science, Adekunle Ajasin

University, Akungba Akoko, Ondo State, Nigeria

\begin{abstract}
This study investigated the antibacterial potential of fermented palm wine tapped from Elaeis guineensis on some selected diarrhoeagenic bacteria which included; Escherichia coli, Staphylococcus aureus, Klebsiella oxytoca, Pseudomonas aeruginosa and Salmonella pullorum. The antibacterial assay of the freshly tapped palm wine was performed using agar well diffusion technique. The succession of inherent microorganisms in the palm wine, the microbial load as well as the $\mathrm{pH}$ of the medium were monitored as fermentation progressed. The microbial flora observed during succession included: Saccharomyces cerevisiae, Bacillus pumilis, Candida kefyr, Clavibacter michiganensis, Coryne bacterium diphtheria, Cellulomonas cellulans, Bacillus subtilis and Mycobacterium agri. The total bacterial count ranged between $6.01 \pm 0.12^{a}$ and $11.8 \pm 0.12^{a} \mathrm{CFU} / \mathrm{ml}$, while the total yeast count ranged between $1.10 \pm 0.10^{b}$ and $4.2 \pm 0.16^{a b} \mathrm{SFU} / \mathrm{ml}$. The undiluted palm wine sample had a growth inhibitory effect on the test organisms with diameter zones of inhibition ranging from $11.00 \pm 1.21^{b}$ to $26.430 \pm$ 3.80'. Palm wine subjected to fermentation for 168 hours exerted the highest inhibitory effect on all the selected diarrhoeagenic bacteria. The inhibition mediated by the palm wine compared favourably with that of some conventional antibiotics employed in this study. It is conceivable that freshly tapped palm wine subjected to natural fermentation could be used to treat diarrhea caused by these bacteria.
\end{abstract}

Keywords: Antibacterial; Diarrhoeagenic; Elaeis guineensis, Fermentation; Palm wine

\section{INTRODUCTION}

Palm wine is an alcoholic beverage produced by natural fermentation of sap of various palms, which include Elaeis guineensis, Raphia regalis, Raphia sudanica, Raphia vinifera, Raphia hookeri and Borassus aethiopum[1].The unfermented sap is a clean, sweet, colourless syrup. It is a refreshing beverage widely consumed in Southern Nigeria and other parts of the world particularly Asia and Southern America [2]. Although palm wine may be presented in a variety of flavours, ranging from sweet (unfermented) to sour (fermented) and vinegary, however, it is mostly enjoyed when sweet [3].

Palm sap has some microflora such as Saccharomyces cerevisiae which is used in the production of acceptable wines from tropical fruits [4], [5]. Palm wine is usually a whitish and effervescent liquid, both properties derive from the fact that the fermented organisms are numerous and alive when the beverage is consumed, it differs from grape wines in that, it is opaque. [6].

Palm wine is normally used traditionally for the extraction of active ingredients from leaves, barks and stems of some medicinal trees used in the treatment of various diseases like malaria, yellow fever and stomach disorders [7]. It is also used to treat cases of skin rashes in children and related diseases like smallpox, chicken pox and measles. It also has religious, social and nutritional use [8]. Palm wine can be used for culinary purposes, for example, it can be used as a yeast substitute for leavening food products. Palm wine is nutritionally important because it is an excellent source of probiotic, nicotinic acid, thiamin, vitamin C, protein and riboflavin [9].

The discovery of new antimicrobial agents from different sources such as microorganisms, animals, plants and plant products has been the major challenge of researchers [4]. The increase in drug resistance by microorganisms, higher cost of commercially produced antimicrobial agents, coupled with development of new strains of microbes adds urgency to the search for cheaper antimicrobial agents [10]. This study therefore investigated the antibacterial potential of palm wine tapped from Elaeis guineensis on selected bacterial etiological agents of diarrhoea. 


\section{MATERIALS AND METHODS}

\subsection{Source of Palm Wine Samples Used}

Freshly top-tapped palm wine samples (Elaeis guineensis) were obtained from a local palm wine tapper in AkungbaAkoko, Ondo state, Nigeria. The samples were collected using sterilized labelled one litre capacity sample bottles with screw caps. This method of collection was according to [11] and [1] which reduce fermentation rate considerably before the palm wine is taken into the laboratory for further studies.

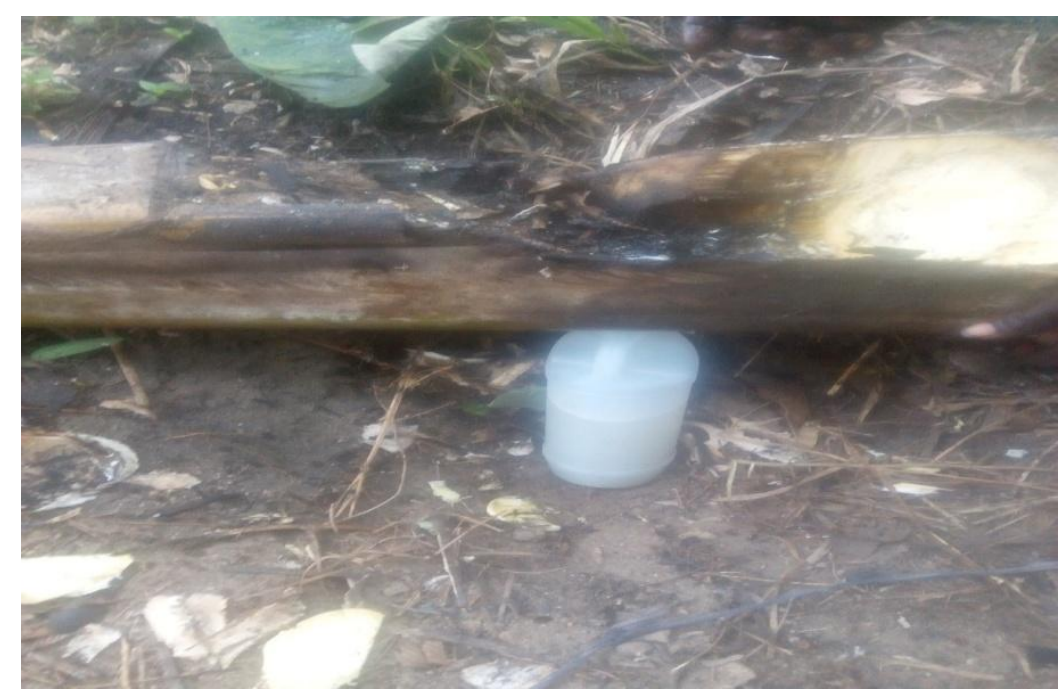

Plate1. Collection of Palm wine sample from Elaeis guineensistree

\subsection{Source of Test Organisms}

Escherichia coli, Salmonella typhi, Staphylococcus aureus, Salmonella pullorum, and Klebsiella oxytoca were collected from AdekunleAjasin University Health Centre and then confirmed using selective media and various biochemical tests to ascertain their identities [12].

\subsection{Standardization of Test Organism}

Cell suspensions of the bacteria to be tested were prepared in sterile saline or Müeller-Hinton broth for 24 hours. The cell suspension was prepared by transferring a portion of the fresh growth with a sterile swab or inoculating loop to the suspending medium, using caution when mixing the cells with the suspending medium to avoid formation of bubbles. The suspension was then adjusted to the 0.5 McFarland turbidity standard [12].

\subsection{Assessment of Growth Inhibitory Activity of Palm Wine on the Test Organisms}

Using the technique of agar well diffusion [13], one milliliter of standardized cells of each of the test organisms was taken using a sterile syringe and placed into sterile petri-dishes (different organism per plate). Each plate was then overlaid with $20 \mathrm{ml}$ nutrient agar, carefully swirled to allow even distribution of the organisms within the agar and allowed to gel before 5 wells $(8 \mathrm{~mm}$ in diameter) were bored in the agar with the aid of a sterile cork borer. $0.1 \mathrm{ml}$ of the undiluted palm wine sample and the ten-fold serially diluted sample $(1: 10,1: 100,1: 1000,1: 10000)$ were dispensed into the wells as appropriate. The plates were incubated at $37^{\circ} \mathrm{C}$ for $24 \mathrm{~h}$. The diameter of the zones of inhibition around the wells containing the palm wine was determined and recorded. This assay was repeated every $24 \mathrm{~h}$ for 7 days to determine the effect of fermentation of palm wine on growth inhibition against the pathogens [12].

\subsection{Antibiotic Sensitivity Test}

The antibiotics used included: Chloramphenicol, Ciprofloxacin, Tetracycline, Ampiclox and Ampicillin. Serial dilution of the test organisms was carried out. Sterilized Mueller-Hinton agar was poured into the plates and allows to gel, wells were made on the plates. Using sterile swab stick, the test organism was used to swab plate. $0.1 \mathrm{ml}$ of the antibiotics which has been dissolved in $820 \mathrm{ml}$ of water $(30 \mu \mathrm{g} / 0.1 \mathrm{ml})$ was then dispensed into the wells and incubated at $37^{\circ} \mathrm{C}$ for 24 hours. Diameter of zones of inhibition was recorded [12]. 


\subsection{Isolation and Characterization of Bacteria and Fungi Present in the Fermented Palm Wine}

One $\mathrm{ml}$ of the palm wine sample was collected aseptically at $0,24,48,72,96,120,144$ and 168 hours of fermentation and serially diluted in sterile peptone water. $0.1 \mathrm{ml}$ aliquots of suitable dilution were inoculated in duplicates by spread plate method on Nutrient agar (NA) for Total Bacterial Count and Potato dextrose agar (PDA) for Total Yeast Count [14]. The inoculated plates were incubated aseptically at $30^{\circ} \mathrm{C}$ for 24 hours for bacteria and $24-48$ hours for the yeast at $22^{\circ} \mathrm{C}$. The recovered isolates were purified by sub-culturing and stored on agar slants at $40 \mathrm{C}$ for characterization.

\section{BIOCHEMICAL TEST}

\subsection{Gram Staining}

A thin smear from each bacterial culture was prepared on clean grease-free slides by dissolving a minute portion of the colony obtained from a 24 hours old culture of each bacterial in one drop of distilled water on the slide. This was subsequently air dried and heat fixed by passing over gentle flame. Each heat-fixed smear was stained by addition of 2 drops of crystal violet solution for $60 \mathrm{sec}$ and rinsed with water. The smear were again flooded with Gram's iodine for $30 \mathrm{sec}$ and rinsed with water, decolorized with $70 \%$ alcohol for $15 \mathrm{sec}$ and were rinsed with distilled water. They were then counter stained with 2 drops of Safranin for $60 \mathrm{sec}$ and finally rinsed with water, then allowed to air dry. The smears were mounted on a Microscope and observed under oil immersion objective lens. Gram negative cells appeared pink or red, while Gram positive organisms appeared purple [15].

\subsection{Motility Test}

This test was done to demonstrate whether isolate was motile or not, culture used for this test was1824 hours old broth culture, then $10 \mathrm{ml}$ of prepared nutrient agar was poured into a universal bottles and allowed to solidify. A sterile syringe was then dipped inside the broth culture of the organisms grown and then used to stab the solidified agar in the middle; the universal bottle was then incubated at $370 \mathrm{C}$ for $24 \mathrm{~h}$ and examined for motile action. If the organism diffused around the agar then it is motile but if there was no diffusion, then the organisms was said to be non-motile [12].

\subsection{Catalase Test}

The test was carried out to detect the presence of Catalase which converts hydrogen peroxide to water and oxygen. A wire loop was used to pick up the organism to be tested from a culture plate and placed in a drop of hydrogen peroxide on a clean glass slide. Formation of gas bubbles indicates a positive reaction, while absence of gas bubble indicates negative reaction [14].

\subsection{Coagulase Test}

A sterile wire loop was used to pick a colony from an overnight culture and mixed with a normal saline placed at the end of a clean glass slide. Drop of blood plasma was added and incubated at 370C for 1-6 hours. Clumping within 1 to 6 hours (1-6hrs) indicates a positive reaction [16].

\subsection{Oxidase Test}

Culture of the bacteria was made on an agar medium and allowed to grow. After growth a freshly prepared $1 \%$ tetra-methyl-p-phenylene-diaminedihydrochloride was poured on the plate so as to cover the surface, this is then decanted. Oxidase positive developed purple color rapidly while Oxidase negative do not develop the purple Color [17].

\subsection{Citrate Test}

This test detects the ability of an organism to utilize citrate as a sole source of carbon and energy. About $2.4 \mathrm{~g}$ of citrate agar was dissolve in $100 \mathrm{~mL}$ of distilled water. About nine milliliter $(9 \mathrm{~mL})$ of citrate medium was dispensed into each tube and covered, then sterilized and allowed to cool in a slanted position. The tubes were inoculated by streaking the organisms once across the surface. A change from green to blue indicates utilization of the citrate [17].

\subsection{Sugar Fermentation Test}

Sugar fermentation test was carried out to determine the ability of organisms to ferment sugars with production of acid and gas. Sugar indicator broth was prepared using peptone water medium containing 1\% sugars (Glucose, Lactose, Maltose, Fructose and Sucrose) and 0.01\% phenol red. 
About nine millimeters of sugar broth was dispensed into each of the test tubes, durham tube which would trap the gas if produced was inverted carefully. The test tubes were autoclaved and inoculated with a loopful of $24 \mathrm{~h}$ old culture of the test organisms after then incubated for $2-7$ days at $36^{\circ} \mathrm{C}$ and observed daily for acid and gas production. Yellow coloration indicates acid production while gas production was indicated by displacement of the medium in the durham tube [17].

\subsection{Indole Test}

Tryptone broth $(5 \mathrm{~mL})$ was placed into different test tubes after which a loopful of the bacterial isolates was inoculated into the test tubes, leaving one of the test tubes uninoculated to serve as control. The test tubes were then incubated at 370C for $48 \mathrm{~h}$. After incubation, 3drops of Kovac's reagent was added and shaken gently; it was allowed to stand for 20 min to permit the reagent to rise. A red colour at the top surface of the tube indicates a positive result while yellow coloration indicates a negative result[14].

\subsection{Methyl Red}

Five millimeters of glucose phosphate broth $(1 \mathrm{~g}$ glucose, $0.5 \% \mathrm{KH} 2 \mathrm{PO} 4,0.5 \%$ peptone and $100 \mathrm{~mL}$ distilled water) were dispensed in clean test tubes and sterilized. The tubes were then inoculated with the test organisms and incubated at $37^{\circ} \mathrm{C}$ for $48 \mathrm{hrs}$. At the end of incubation, few drops of methyl red solution were added to each test and colour change was observed. A red colour indicates a positive reaction.

\subsection{Determination of $\mathbf{p H}$}

$\mathrm{pH}$ was measured using digital $\mathrm{pH}$ meter after homogenizing $5 \mathrm{ml}$ of the palm wine samples in $40 \mathrm{ml}$ of distilled water. The $\mathrm{pH}$ meter was standardized with buffer solution; the buffer solution was prepared with $\mathrm{pH}$ buffer powder of $\mathrm{pH} 4.00$ at ${ }^{0}$ which is dissolved in $250 \mathrm{ml}$ distilled water. The electrode of the $\mathrm{pH}$ meter was immersed in a glass beaker containing the sample; Readings were obtained from the photo-detector of the $\mathrm{pH}$ meter [12].

\subsection{Total Titratable Acid}

This was determined using the method of [18], 10ml of the fermenting medium was transferred into a beaker, followed by the addition of 3 drops of phenolphthalein indicator. The sample was then titrated against $0.1 \mathrm{M} \mathrm{NaOH}$ to an end point of a definite pink colour. The volume of $\mathrm{NaOH}$ used was noted and the titratable acid percentage was calculated using the following formula;

TTA $(\%)=\mathrm{V} \times 0.15$

Where; $\mathrm{V}=$ Volume of $\mathrm{NaOH}$.

\subsection{Statistical Analysis}

Data obtained were subjected to one way analysis of variance test using SPSS 25.0, software (SPSS Inc., Chicago, IL,USA), the means were compared using Duncan's new Multiple range test. The results were expressed as Mean \pm Standard Deviation (SD), where the level of significance was considered at $\mathrm{P}<0.05$.

\section{RESUlT}

The result of the growth inhibitory effect of undiluted palm wine against the selected diarrhoeagenic bacteria is presented in Table 1. The zone of inhibition against the test isolates increased significantly $(\mathrm{p}<0.05)$ in Days 5, 6 and 7 when compared with other days. While in the groups of antibiotics, Chloramphenicol, Tetracycline and Ciprofloxacin were observed to exhibit significant $(\mathrm{p}<0.05)$ zones of inhibition against the test isolates when compared with others.

Table1. Growth inhibitory effect of undiluted palm wine on selected diarrhoeagenic bacteria.

\begin{tabular}{|c|c|c|c|c|c|}
\hline \multicolumn{6}{|c|}{ Diameters of Zones of Inhibition (mm) } \\
\hline $\begin{array}{ll}\text { Days } & \text { Of } \\
\text { Fermentation } & \end{array}$ & $\begin{array}{l}\text { Escherichia } \\
\text { Coli }\end{array}$ & $\begin{array}{l}\text { Staphylococcus } \\
\text { Aureus }\end{array}$ & $\begin{array}{l}\text { Pseudomonas } \\
\text { Aeruginosa }\end{array}$ & $\begin{array}{l}\text { Klebsiellaox } \\
\text { ytoca }\end{array}$ & $\begin{array}{l}\text { Salmonella } \\
\text { Pullorum }\end{array}$ \\
\hline Day 1 & $13.00 \pm 1.95^{\mathrm{b}}$ & $22.00 \pm 3.30^{\mathrm{b}}$ & $13.00 \pm 1.95^{\mathrm{b}}$ & $0.00 \pm 0.00^{\mathrm{b}}$ & $0.00 \pm 0.00^{\mathrm{a}}$ \\
\hline Day 2 & $11.90 \pm 0.00^{\mathrm{a}}$ & $20.00 \pm 3.00^{\mathrm{b}}$ & $17.00 \pm 2.55^{\mathrm{bc}}$ & $0.00 \pm 0.00^{\mathrm{a}}$ & $0.00 \pm 0.00^{\mathrm{a}}$ \\
\hline Day 3 & $19.00 \pm 2.85^{\mathrm{c}}$ & $19.00 \pm 5.11^{\mathrm{a}}$ & $18.12 \pm 0.00^{\mathrm{a}}$ & $0.00 \pm 0.00^{\mathrm{a}}$ & $0.00 \pm 0.00^{\mathrm{a}}$ \\
\hline Day 4 & $22.05 \pm 0.95^{\mathrm{a}}$ & $25.00 \pm 3.75^{\mathrm{bc}}$ & $18.79 \pm 0.05^{\mathrm{a}}$ & $0.00 \pm 0.00^{\mathrm{a}}$ & $0.00 \pm 0.00^{\mathrm{b}}$ \\
\hline Day 5 & $25.10 \pm 4.50^{\mathrm{d}}$ & $26.430 \pm 3.80^{\mathrm{c}}$ & $25.80 \pm 0.90^{\mathrm{d}}$ & $0.00 \pm 0.00^{\mathrm{a}}$ & $0.00 \pm 0.00^{\mathrm{a}}$ \\
\hline Day 6 & $20.00 \pm 4.80^{\mathrm{c}}$ & $24.92 \pm 5.60^{\mathrm{c}}$ & $20.00 \pm 2.50^{\mathrm{c}}$ & $0.00 \pm 0.00^{\mathrm{a}}$ & $0.00 \pm 0.00^{\mathrm{a}}$ \\
\hline
\end{tabular}


Therapeutic Potential of Palm Wine on Selected Diarrhoeagenic Bacteria

\begin{tabular}{|l|l|l|l|l|l|}
\hline Day 7 & $20.00 \pm 3.00^{\mathrm{c}}$ & $21.00 \pm 3.15^{\mathrm{bc}}$ & $20.00 \pm 3.00^{\mathrm{c}}$ & $22.00 \pm 3.30^{\mathrm{c}}$ & $18.00 \pm 2.70^{\mathrm{bc}}$ \\
\hline Ampiclox & $0.00 \pm 0.00 \mathrm{a}$ & $17.00 \pm 2.55^{\mathrm{b}}$ & $17.00 \pm 2.55^{\mathrm{bc}}$ & $0.00 \pm 0.00^{\mathrm{a}}$ & $0.00 \pm 0.00^{\mathrm{a}}$ \\
\hline Ampicillin & $0.00 \pm 0.00 \mathrm{a}$ & $20.00 \pm 3.00^{\mathrm{b}}$ & $20.00 \pm 3.00^{\mathrm{c}}$ & $0.00 \pm 0.00^{\mathrm{a}}$ & $0.00 \pm 0.00^{\mathrm{a}}$ \\
\hline Chloramphenicol & $0.00 \pm 0.00 \mathrm{a}$ & $26.00 \pm 3.90^{\mathrm{bc}}$ & $26.00 \pm 3.90^{\mathrm{cd}}$ & $20.00 \pm 3.00^{\mathrm{c}}$ & $30.00 \pm 4.50^{\mathrm{d}}$ \\
\hline Tetracycline & $17.00 \pm 2.55^{\mathrm{bc}}$ & $22.00 \pm 3.30^{\mathrm{bc}}$ & $22.00 \pm 3.30^{\mathrm{c}}$ & $23.00 \pm 3.45^{\mathrm{c}}$ & $19.00 \pm 2.85^{\mathrm{c}}$ \\
\hline Ciprofloxacin & $17.00 \pm 1.86^{\mathrm{bc}}$ & $27.00 \pm 4.05^{\mathrm{bc}}$ & $27.00 \pm 4.05^{\mathrm{cd}}$ & $21.00 \pm 3.15^{\mathrm{c}}$ & $14.00 \pm 2.10^{\mathrm{b}}$ \\
\hline
\end{tabular}

Values are mean \pm S.D., $n=3$, Means with different superscripts down the column are significantly different at $P<0.05$

For the serially diluted palmwine 1:10,zone of inhibition observed against Escherichia coli was significant on Day 5, while no zone of inhibition was recorded against Pseudomonas aeruginosa, Klebsiella oxytoca and Salmonella pullorumas presented in Table 2.

Table2. Growth inhibitory effect of diluted palm wine (1:10) on selected diarrhoeagenic bacteria.

\begin{tabular}{|l|l|l|l|l|l|}
\hline \multicolumn{7}{|c|}{ Diameters of Zones of Inhibition (mm) } \\
\hline $\begin{array}{l}\text { Days Of } \\
\text { Fermentation }\end{array}$ & Escherichia coli & $\begin{array}{l}\text { Staphylococcus } \\
\text { aureus }\end{array}$ & $\begin{array}{l}\text { Pseudomonas } \\
\text { aeruginosa }\end{array}$ & $\begin{array}{l}\text { Klebsiellaoxy } \\
\text { toca }\end{array}$ & $\begin{array}{l}\text { Salmonella } \\
\text { pullorum }\end{array}$ \\
\hline Day 1 & $11.00 \pm 1.65^{\mathrm{b}}$ & $0.00 \pm 0.00^{\mathrm{a}}$ & $0.00 \pm 0.00^{\mathrm{a}}$ & $0.00 \pm 0.00^{\mathrm{a}}$ & $0.00 \pm 0.00^{\mathrm{a}}$ \\
\hline Day 2 & $9.50 \pm 2.00^{\mathrm{a}}$ & $00.00 \pm 0.0^{\mathrm{b}}$ & $0.00 \pm 0.00^{\mathrm{a}}$ & $0.00 \pm 0.00^{\mathrm{a}}$ & $0.00 \pm 0.00^{\mathrm{a}}$ \\
\hline Day 3 & $17.00 \pm 2.55^{\mathrm{c}}$ & $0.00 \pm 0.00^{\mathrm{a}}$ & $0.00 \pm 0.00^{\mathrm{a}}$ & $0.00 \pm 0.00^{\mathrm{a}}$ & $0.00 \pm 0.00^{\mathrm{a}}$ \\
\hline Day 4 & $18.00 \pm 0.04^{\mathrm{a}}$ & $0.00 \pm 0.00^{\mathrm{a}}$ & $0.00 \pm 0.00^{\mathrm{a}}$ & $0.00 \pm 0.00^{\mathrm{a}}$ & $0.00 \pm 0.00^{\mathrm{a}}$ \\
\hline Day 5 & $25.00 \pm 3.75^{\mathrm{d}}$ & $0.00 \pm 0.00^{\mathrm{a}}$ & $0.00 \pm 0.00^{\mathrm{a}}$ & $0.00 \pm 0.00^{\mathrm{a}}$ & $0.00 \pm 0.00^{\mathrm{a}}$ \\
\hline Day 6 & $15.00 \pm 2.25^{\mathrm{c}}$ & $0.00 \pm 0.00^{\mathrm{a}}$ & $0.00 \pm 0.00^{\mathrm{a}}$ & $0.00 \pm 0.00^{\mathrm{a}}$ & $0.00 \pm 0.00^{\mathrm{a}}$ \\
\hline Day 7 & $16.00 \pm 2.40^{\mathrm{c}}$ & $0.00 \pm 0.00^{\mathrm{a}}$ & $0.00 \pm 0.00^{\mathrm{a}}$ & $0.00 \pm 0.00^{\mathrm{a}}$ & $0.00 \pm 0.00^{\mathrm{a}}$ \\
\hline Ampiclox & $0.00 \pm 0.00^{\mathrm{a}}$ & $17.00 \pm 2.55^{\mathrm{b}}$ & $17.00 \pm 2.55^{\mathrm{b}}$ & $0.00 \pm 0.00^{\mathrm{a}}$ & $0.00 \pm 0.00^{\mathrm{a}}$ \\
\hline Ampicillin & $0.00 \pm 0.00^{\mathrm{a}}$ & $20.00 \pm 3.00^{\mathrm{b}}$ & $20.00 \pm 3.00^{\mathrm{bc}}$ & $0.00 \pm 0.00^{\mathrm{a}}$ & $0.00 \pm 0.00^{\mathrm{a}}$ \\
\hline Chloramphenicol & $0.00 \pm 0.00^{\mathrm{a}}$ & $26.00 \pm 3.90^{\mathrm{c}}$ & $26.00 \pm 3.90^{\mathrm{c}}$ & $20.00 \pm 3.00^{\mathrm{b}}$ & $30.00 \pm 4.50^{\mathrm{d}}$ \\
\hline Tetracycline & $17.00 \pm 2.55^{\mathrm{c}}$ & $22.00 \pm 3.30^{\mathrm{b}}$ & $22.00 \pm 3.30^{\mathrm{bc}}$ & $23.00 \pm 3.45^{\mathrm{b}}$ & $19.00 \pm 2.85^{\mathrm{c}}$ \\
\hline Ciprofloxacin & $17.00 \pm 1.86^{\mathrm{c}}$ & $27.00 \pm 4.05^{\mathrm{c}}$ & $27.00 \pm 4.05^{\mathrm{c}}$ & $21.00 \pm 3.15^{\mathrm{b}}$ & $14.00 \pm 2.10^{\mathrm{b}}$ \\
\hline
\end{tabular}

Values are mean \pm S.D., $n=3$, Means with different superscripts down the column are significantly different at $P<0.05$

Table 3 shows the growth inhibitory effect of diluted palm wine (1:100) on the selected pathogens. Zones of inhibition against Escherichia coli, Staphylococcus aureus and Pseudomonas aeruginosa was observed on Day 7.There was no zone of inhibition recorded for Klebsiella oxytoca and Salmonella pullorum from Day 1 to Day 7.

Table3. Growth inhibitory effect of diluted palm wine (1:100) on selected diarrhoeagenic bacteria.

\begin{tabular}{|l|l|l|l|l|l|}
\hline Diameters of Zones of Inhibition (mm) \\
\hline Days Of Fermentation & $\begin{array}{l}\text { Escherichia } \\
\text { coli }\end{array}$ & $\begin{array}{l}\text { Staphylococcu } \\
\text { saureus }\end{array}$ & $\begin{array}{l}\text { Pseudomonas } \\
\text { aeruginosa }\end{array}$ & $\begin{array}{l}\text { Klebsiellaoxy } \\
\text { toca }\end{array}$ & $\begin{array}{l}\text { Salmonella } \\
\text { pullorum }\end{array}$ \\
\hline Day 1 & $0.00 \pm 0.00^{\mathrm{a}}$ & $0.00 \pm 0.00^{\mathrm{a}}$ & $0.00 \pm 0.00^{\mathrm{a}}$ & $0.00 \pm 0.00^{\mathrm{a}}$ & $0.00 \pm 0.00^{\mathrm{a}}$ \\
\hline Day 2 & $0.00 \pm 0.00^{\mathrm{a}}$ & $0.00 \pm 0.00^{\mathrm{b}}$ & $0.00 \pm 0.00^{\mathrm{a}}$ & $0.00 \pm 0.00^{\mathrm{a}}$ & $0.00 \pm 0.00^{\mathrm{a}}$ \\
\hline Day 3 & $0.00 \pm 0.00^{\mathrm{a}}$ & $0.00 \pm 0.00^{\mathrm{a}}$ & $0.00 \pm 0.00^{\mathrm{a}}$ & $0.00 \pm 0.00^{\mathrm{a}}$ & $0.00 \pm 0.00^{\mathrm{a}}$ \\
\hline Day 4 & $0.00 \pm 0.00^{\mathrm{a}}$ & $0.00 \pm 0.00^{\mathrm{a}}$ & $0.00 \pm 0.00^{\mathrm{a}}$ & $0.00 \pm 0.00^{\mathrm{a}}$ & $0.00 \pm 0.00^{\mathrm{a}}$ \\
\hline Day 5 & $0.00 \pm 0.00^{\mathrm{a}}$ & $0.00 \pm 0.00^{\mathrm{a}}$ & $0.00 \pm 0.00^{\mathrm{a}}$ & $0.00 \pm 0.00^{\mathrm{a}}$ & $0.00 \pm 0.00^{\mathrm{a}}$ \\
\hline Day 6 & $0.00 \pm 0.00^{\mathrm{a}}$ & $0.00 \pm 0.00^{\mathrm{a}}$ & $0.00 \pm 0.00^{\mathrm{a}}$ & $0.00 \pm 0.00^{\mathrm{a}}$ & $0.00 \pm 0.00^{\mathrm{a}}$ \\
\hline Day 7 & $13.0 \pm 0.95^{\mathrm{b}}$ & $12.00 \pm 1.80^{\mathrm{b}}$ & $15.00 \pm 2.25^{\mathrm{b}}$ & $0.00 \pm 0.00^{\mathrm{a}}$ & $0.00 \pm 0.00^{\mathrm{a}}$ \\
\hline Ampiclox & $0.00 \pm 0.00^{\mathrm{a}}$ & $17.00 \pm 2.55^{\mathrm{c}}$ & $17.00 \pm 2.55^{\mathrm{b}}$ & $0.00 \pm 0.00^{\mathrm{a}}$ & $0.00 \pm 0.00^{\mathrm{a}}$ \\
\hline Ampicillin & $0.00 \pm 0.00^{\mathrm{a}}$ & $20.00 \pm 3.00^{\mathrm{cd}}$ & $20.00 \pm 3.00^{\mathrm{bc}}$ & $0.00 \pm 0.00^{\mathrm{a}}$ & $0.00 \pm 0.00^{\mathrm{a}}$ \\
\hline Chloramphenicol & $0.00 \pm 0.00^{\mathrm{a}}$ & $26.00 \pm 3.90^{\mathrm{d}}$ & $26.00 \pm 3.90^{\mathrm{c}}$ & $20.00 \pm 3.00^{\mathrm{b}}$ & $30.00 \pm 4.50^{\mathrm{d}}$ \\
\hline Tetracycline & $17.00 \pm 2.55^{\mathrm{c}}$ & $22.00 \pm 3.30^{\mathrm{cd}}$ & $22.00 \pm 3.30^{\mathrm{c}}$ & $23.00 \pm 3.45^{\mathrm{b}}$ & $19.00 \pm 2.85^{\mathrm{c}}$ \\
\hline Ciprofloxacin & $17.00 \pm 1.86^{\mathrm{c}}$ & $27.00 \pm 4.05^{\mathrm{d}}$ & $27.00 \pm 4.05^{\mathrm{cd}}$ & $21.00 \pm 3.15^{\mathrm{b}}$ & $14.00 \pm 2.10^{\mathrm{b}}$ \\
\hline
\end{tabular}

Values are mean \pm S.D., $n=3$, Means with different superscripts down the column are significantly different at $P<0.05$

The growth inhibitory effects of the1:1000 and1:10000diluted palm wine on the selected pathogens are shown in Tables 4 and 5 respectively. Zones of inhibition against Escherichia coli and Staphylococcus aureus were recorded on Day 7.There was no zone of inhibition recorded against Pseudomonas aeruginosa, Klebsiella oxytoca and Salmonella pullorum from Day 1 to Day 7. 
Therapeutic Potential of Palm Wine on Selected Diarrhoeagenic Bacteria

Table4. Growth inhibitory effect of diluted palm wine (1:1000) on selected diarrhoeagenic bacteria.

\begin{tabular}{|c|c|c|c|c|c|}
\hline \multicolumn{6}{|c|}{ Diameters of Zones of Inhibition (mm) } \\
\hline $\begin{array}{ll}\text { Days } & \text { Of } \\
\text { Fermentation } & \\
\end{array}$ & $\begin{array}{l}\text { Escherichia } \\
\text { Coli }\end{array}$ & $\begin{array}{l}\text { Staphylococcu } \\
\text { s Aureus }\end{array}$ & $\begin{array}{l}\text { Pseudomonas } \\
\text { Aeruginosa }\end{array}$ & $\begin{array}{l}\text { Klebsiellaoxy } \\
\text { toca }\end{array}$ & $\begin{array}{l}\text { Salmonella } \\
\text { Pullorum }\end{array}$ \\
\hline Day 1 & $0.00 \pm 0.00^{\mathrm{a}}$ & $0.00 \pm 0.00^{\mathrm{a}}$ & $0.00 \pm 0.00^{\mathrm{a}}$ & $0.00 \pm 0.00^{\mathrm{a}}$ & $0.00 \pm 0.00^{\mathrm{a}}$ \\
\hline Day 2 & $0.00 \pm 0.00^{\mathrm{a}}$ & $0.00 \pm 0.00^{\mathrm{a}}$ & $0.00 \pm 0.00^{\mathrm{a}}$ & $0.00 \pm 0.00^{\mathrm{a}}$ & $0.00 \pm 0.00^{\mathrm{a}}$ \\
\hline Day 3 & $0.00 \pm 0.00^{\mathrm{b}}$ & $0.00 \pm 0.00^{\mathrm{a}}$ & $0.00 \pm 0.00^{\mathrm{a}}$ & $0.00 \pm 0.00^{\mathrm{a}}$ & $0.00 \pm 0.00^{\mathrm{a}}$ \\
\hline Day 4 & $0.00 \pm 0.00^{\mathrm{a}}$ & $0.00 \pm 0.00^{\mathrm{a}}$ & $0.00 \pm 0.00^{\mathrm{a}}$ & $0.00 \pm 0.00^{\mathrm{a}}$ & $0.00 \pm 0.00^{\mathrm{a}}$ \\
\hline Day 5 & $0.00 \pm 0.00^{\mathrm{a}}$ & $0.00 \pm 0.00^{\mathrm{a}}$ & $0.00 \pm 0.00^{\mathrm{a}}$ & $0.00 \pm 0.00^{\mathrm{a}}$ & $0.00 \pm 0.00^{\mathrm{a}}$ \\
\hline Day 6 & $0.00 \pm 0.00^{\mathrm{a}}$ & $0.00 \pm 0.00^{\mathrm{a}}$ & $0.00 \pm 0.00^{\mathrm{a}}$ & $0.00 \pm 0.00^{\mathrm{a}}$ & $0.00 \pm 0.00^{\mathrm{a}}$ \\
\hline Day 7 & $12.00 \pm 2.52^{\mathrm{b}}$ & $11.00 \pm 1.21^{\mathrm{b}}$ & $0.00 \pm 0.00^{\mathrm{a}}$ & $0.00 \pm 0.00^{\mathrm{a}}$ & $0.00 \pm 0.00^{\mathrm{a}}$ \\
\hline Ampiclox & $0.00 \pm 0.00^{\mathrm{a}}$ & $17.00 \pm 2.55^{\mathrm{b}}$ & $17.00 \pm 2.55^{\mathrm{b}}$ & $0.00 \pm 0.00^{\mathrm{a}}$ & $0.00 \pm 0.00^{\mathrm{a}}$ \\
\hline Ampicillin & $0.00 \pm 0.00^{\mathrm{a}}$ & $20.00 \pm 3.00^{\mathrm{bc}}$ & $20.00 \pm 3.00^{\mathrm{b}}$ & $0.00 \pm 0.00^{\mathrm{a}}$ & $0.00 \pm 0.00^{\mathrm{a}}$ \\
\hline Chloramphenicol & $0.00 \pm 0.00^{\mathrm{a}}$ & $26.00 \pm 3.90^{\mathrm{c}}$ & $26.00 \pm 3.90^{\mathrm{c}}$ & $20.00 \pm 3.00^{\mathrm{b}}$ & $30.00 \pm 4.50^{\mathrm{d}}$ \\
\hline Tetracycline & $17.00 \pm 2.55^{\mathrm{bc}}$ & $22.00 \pm 3.30^{\mathrm{bc}}$ & $22.00 \pm 3.30^{\mathrm{bc}}$ & $23.00 \pm 3.45^{\mathrm{b}}$ & $19.00 \pm 2.85^{\mathrm{c}}$ \\
\hline Ciprofloxacin & $17.00 \pm 1.86^{\mathrm{bc}}$ & $27.00 \pm 4.05^{\mathrm{c}}$ & $27.00 \pm 4.05^{\mathrm{c}}$ & $21.00 \pm 3.15^{\mathrm{b}}$ & $14.00 \pm 2.10^{\mathrm{b}}$ \\
\hline
\end{tabular}

Values are mean \pm S.D., $n=3$, Means with different superscripts down the column are significantly different at $P<0.05$

Table5. Growth inhibitory effect of diluted palm wine (1:10000) on selected diarrhoeagenic bacteria.

\begin{tabular}{|l|l|l|l|l|l|}
\hline \multicolumn{7}{|c|}{ Diameters of Zones of Inhibition (mm) } \\
\hline $\begin{array}{l}\text { Days Of } \\
\text { Fermentation }\end{array}$ & $\begin{array}{l}\text { Escherichia } \\
\text { Coli }\end{array}$ & $\begin{array}{l}\text { Staphylococcus } \\
\text { Aureus }\end{array}$ & $\begin{array}{l}\text { Pseudomonas } \\
\text { Aeruginosa }\end{array}$ & $\begin{array}{l}\text { Klebsiellaoxy } \\
\text { toca }\end{array}$ & $\begin{array}{l}\text { Salmonella } \\
\text { Pullorum }\end{array}$ \\
\hline Day 1 & $0.00 \pm 0.00^{\mathrm{a}}$ & $0.00 \pm 0.00^{\mathrm{a}}$ & $0.00 \pm 0.00^{\mathrm{a}}$ & $0.00 \pm 0.00^{\mathrm{a}}$ & $0.00 \pm 0.00^{\mathrm{a}}$ \\
\hline Day 2 & $0.00 \pm 0.00^{\mathrm{a}}$ & $0.00 \pm 0.00^{\mathrm{a}}$ & $0.00 \pm 0.00^{\mathrm{a}}$ & $0.00 \pm 0.00^{\mathrm{a}}$ & $0.00 \pm 0.00^{\mathrm{a}}$ \\
\hline Day 3 & $0.00 \pm 0.00^{\mathrm{b}}$ & $0.00 \pm 0.00^{\mathrm{a}}$ & $0.00 \pm 0.00^{\mathrm{a}}$ & $0.00 \pm 0.00^{\mathrm{a}}$ & $0.00 \pm 0.00^{\mathrm{a}}$ \\
\hline Day 4 & $0.00 \pm 0.00^{\mathrm{a}}$ & $0.00 \pm 0.00^{\mathrm{a}}$ & $0.00 \pm 0.00^{\mathrm{a}}$ & $0.00 \pm 0.00^{\mathrm{a}}$ & $0.00 \pm 0.00^{\mathrm{a}}$ \\
\hline Day 5 & $0.00 \pm 0.00^{\mathrm{a}}$ & $0.00 \pm 0.00^{\mathrm{a}}$ & $0.00 \pm 0.00^{\mathrm{a}}$ & $0.00 \pm 0.00^{\mathrm{a}}$ & $0.00 \pm 0.00^{\mathrm{a}}$ \\
\hline Day 6 & $0.00 \pm 0.00^{\mathrm{a}}$ & $0.00 \pm 0.00^{\mathrm{a}}$ & $0.00 \pm 0.00^{\mathrm{a}}$ & $0.00 \pm 0.00^{\mathrm{a}}$ & $0.00 \pm 0.00^{\mathrm{a}}$ \\
\hline Day 7 & $12.00 \pm 1.92^{\mathrm{b}}$ & $11.00 \pm 1.76^{\mathrm{b}}$ & $0.00 \pm 0.00^{\mathrm{a}}$ & $0.00 \pm 0.00^{\mathrm{a}}$ & $0.00 \pm 0.00^{\mathrm{a}}$ \\
\hline Ampiclox & $0.00 \pm 0.00^{\mathrm{a}}$ & $17.00 \pm 2.55^{\mathrm{c}}$ & $17.00 \pm 2.55^{\mathrm{b}}$ & $0.00 \pm 0.00^{\mathrm{a}}$ & $0.00 \pm 0.00^{\mathrm{a}}$ \\
\hline Ampicillin & $0.00 \pm 0.00^{\mathrm{a}}$ & $20.00 \pm 3.00^{\mathrm{c}}$ & $20.00 \pm 3.00^{\mathrm{bc}}$ & $0.00 \pm 0.00^{\mathrm{a}}$ & $0.00 \pm 0.00^{\mathrm{a}}$ \\
\hline Chloramphenicol & $0.00 \pm 0.00^{\mathrm{a}}$ & $26.00 \pm 3.90^{\mathrm{d}}$ & $26.00 \pm 3.90^{\mathrm{c}}$ & $20.00 \pm 3.00^{\mathrm{b}}$ & $30.00 \pm 4.50^{\mathrm{d}}$ \\
\hline Tetracycline & $17.00 \pm 2.55^{\mathrm{c}}$ & $22.00 \pm 3.30^{\mathrm{c}}$ & $22.00 \pm 3.30^{\mathrm{bc}}$ & $23.00 \pm 3.45^{\mathrm{b}}$ & $19.00 \pm 2.85^{\mathrm{c}}$ \\
\hline Ciprofloxacin & $17.00 \pm 1.86^{\mathrm{c}}$ & $27.00 \pm 4.05^{\mathrm{d}}$ & $27.00 \pm 4.05^{\mathrm{c}}$ & $21.00 \pm 3.15^{\mathrm{b}}$ & $14.00 \pm 2.10^{\mathrm{b}}$ \\
\hline
\end{tabular}

Values are mean \pm S.D., $n=3$, Means with different superscripts down the column are significantly different at $P<0.05$

Table6. Succession of the isolated microorganism palm wine during fermentation for 168 hours

\begin{tabular}{|l|l|}
\hline \multicolumn{1}{|c|}{ Duration Of Fermentations } & \multicolumn{1}{c|}{ Types Of Microorganism } \\
\hline Day 1 & Saccharomyces Cerevisiae, Bacillus Pumilis \\
\hline Day 2 & Candida Kefyr, Clavibactermichiganensis \\
\hline Day 3 & Saccharomyces Cerevisiae, Corynebacteriumdiphtheria \\
\hline Day 4 & Saccharomyces Cerevisiae, Cellulomonascellulans, Bacillus Subtilis \\
\hline Day 5 & Saccharomyces Cerevisiae, Bacillus Subtilis, Bacillus Sp. \\
\hline Day 6 & Saccharomyces Cerevisiae, Mycobacterium Agri \\
\hline Day 7 & Saccharomyces Cerevisiae, Microbacteriumlacticum \\
\hline
\end{tabular}

Table7. Physicochemical parameters of the Palm wine during fermentation

\begin{tabular}{|l|l|l|}
\hline \multicolumn{1}{|c|}{ Days Of Fermentation } & \multicolumn{1}{|c|}{ Ph } & Total Titratable Acidity \\
\hline Day 1 & 4.8 & 0.14 \\
\hline Day 2 & 4.0 & 0.18 \\
\hline Day 3 & 3.8 & 0.23 \\
\hline Day 4 & 3.0 & 0.53 \\
\hline Day 5 & 3.0 & 0.91 \\
\hline Day 6 & 2.8 & 1.38 \\
\hline Day 7 & 2.6 & 1.50 \\
\hline
\end{tabular}


Table8. Microbial load of the Palm wine during fermentation

\begin{tabular}{|l|l|l|}
\hline \multicolumn{1}{|c|}{ Days Of Fermentation } & \multicolumn{1}{|c|}{ Bacterial load $(\mathbf{C f u} / \mathbf{m l})$} & \multicolumn{1}{c|}{ Fungi load $(\mathbf{S f u} / \mathbf{m l})$} \\
\hline Day 1 & $11.8 \pm 0.12^{\mathrm{a}}$ & $2.1 \pm 0.14^{\mathrm{bc}}$ \\
\hline Day 2 & $10.2 \pm 0.10^{\mathrm{bc}}$ & $3.2 \pm 0.20^{\mathrm{c}}$ \\
\hline Day 3 & $7.8 \pm 0.40^{\mathrm{c}}$ & $3.8 \pm 0.30^{\mathrm{d}}$ \\
\hline Day 4 & $7.2 \pm 0.08^{\mathrm{a}}$ & $4.2 \pm 0.16^{\mathrm{ab}}$ \\
\hline Day 5 & $8.0 \pm 0.00^{\mathrm{d}}$ & $1.10 \pm 0.10^{\mathrm{b}}$ \\
\hline Day 6 & $7.6 \pm 0.09^{\mathrm{b}}$ & $2.3 \pm 0.20^{\mathrm{d}}$ \\
\hline Day 7 & $6.01 \pm 0.12^{\mathrm{a}}$ & $2.80 \pm 0.27^{\mathrm{a}}$ \\
\hline
\end{tabular}

Values are mean \pm S.D., $n=3$, Means with different superscripts down the column are significantly different at $P<0.05$

\section{DisCUSSION}

This study evaluated the antibacterial potential of palm wine obtained from Elaeis guineensis on some selected diarrhoeagenic bacteria. The trend observed in the level of the microbial load could be associated with the progressive decrease in $\mathrm{pH}$ of the palm wine and changes in the physicochemical quality as fermentation progressed [12].Succession of microbial species depends on various intrinsic and extrinsic factors related to the food matrix including any microbial interactions [19].Diversity in microbial population was observed as fermentation of the palm wine progressed, however, Saccharomyces cerevisiae was found to be most constant all through the fermentation period. This is in coherence with the studies of [20]; [21] and [22], who reported the dominance of Saccharomyces cerevisiae in the fermentation of palm wine. As fermentation progresses and the acid and alcohol contents increase, the more stress tolerant species take over resulting in fewer species completing the fermentation [23]. Some yeast have been noted to be beneficial by producing essential compounds such as folate, degrading toxic compounds such as linamarin, preventing uptake of toxins such as aflatoxin B1 in the human GIT and providing probiotic properties [24].

The variation in zones of inhibition observed for the different palm wine samples across the period of fermentation could be as a result of change in microbial activities as succession occurs.

The undiluted palm wine inhibited the growth of all selected test organisms and exhibited higher zones of inhibition compared to the serially diluted samples. This could be as a result of the activities of the array of microorganisms present which metabolize the sugars present in the palm wine to produce alcohol and organic acid with a consequent decrease in $\mathrm{pH}$ of the sample.

Another factor which could be responsible for the inhibitory effect of the palm wine is the production of bacteriocins by the organisms involved in succession during the fermentation process, bacteriocins have been reported to have inhibitory effects on the growth of microbes [3]; [25]. Some bacteriocins bind to specific receptors on the cell envelope of sensitive target bacteria and cause cell lysis, attack specific intracellular sites such as ribosomes, or disrupt energy production [26].

\section{CONClusion}

This study established that palm wine possess antibacterial activity against selected diarrhoeagenic bacteria. Moreover, fermentation duration plays significant role in the antibacterial activity. The longer the duration of fermentation, the more effective the palm wine in inhibiting the growth of these test organisms. Since palm wine has potent antibacterial activity on the test bacteria, it can be used as an alternative agent for the control of the diarrhoea caused by these organisms in the absence of antibiotics.

\section{REFERENCES}

[1] Obire, O. (2005). Activity of Zygomonas species in palm sap obtained from three areas in Edo State, Nigeria. Journal of Applied Science, Environmental Management. 9:25-30.

[2] Nwokeke, N.V, (2001). Palm wine Preservation Using Traditional Plants that have preservative bases. B.Sc. Thesis. Imo State University, Owerri, Nigeria.

[3] Chung, H.J. and Yousef, A.E (2003). Lactobacillus curvatus produce a bacteriocin-like agent active against gram negative pathogenic bacteria. Journal of Food safety, 25 (2): 59-69.

[4] Cassell GH, Mekalanos J (2001). Development of antimicrobial agents in the era of new and reemerging infectious diseases and increasing antibiotic resistance. Jama. 285(5):601-605.

[5] Obisanya, M. O. Aina, J. O. and Oguntimehin, G. B. (1987). "Production of wine from mango using Saccharomyces and Schizosaccharomyces species isolated from palm wine". Journal of Applied Bacteriology. 63: 191-196. of Biotechnology, 9(27): 4241-4246. 
[6] Okafor, $N$ (1972). The source of the microorganisms in palm wine. Symposium in Nigeria Society of Microbiology 1: 97.

[7] Nitta T, Arai T, Takamatsu H, Inatomi Y, Murata H, Iinuma M, Nakanishi T (2002). Antibacterial activity of extracts prepared from tropical and subtropical plants on methicillin-resistant Staphylococcus aureus. Journal of Health Science. 48(3):273-276.

[8] Uzogara S.G, Agu L.N, Uzogara E .O. (1990). A review of traditional fermented food condiments and beverages in Nigeria. Their Benefits and possible problems. Ecology of Food Nutrient. 24: 267-288.

[9] Okafor, N. (1978).The biochemistry and Microbiology of palm wine. Advances in Applied Microbiology. 24: 237-256.

[10] Kohanski MA, Dwyer DJ, Collins JJ (2010). How antibiotics kill bacteria: From targets to networks. Nature Reviews Microbiology. 8(6):423.

[11] Bassir,O. (1982). Observation of the Fermentation of Palm wine. West African Journal Biology, Applied Chemistry.10: (2) $42-45$.

[12] Akinrotoye KP (2014). Effects of fermented palm wine on some diarrhoeagenic bacteria. Elite Research Journal of Biotechnology and Microbiology. 2:4-14.

[13] Adebolu, T.T and Adaramola, O.B. (2010). Evaluation of growth inhibitory activity of maize "ogi" subjected to continuous and discontinuous fermentation on selected diarrhoeal bacteria. Nigerian journal of biotechnology, 24 (1): 2129-2133.

[14] Cheesbrough, M. (2006). District laboratory practice in tropical countries. Cambridge university press.

[15] Nezuami, N. (2014): elementary microbiology of community health and other allied health practitioners. First published O.J computer Pp. 14-15.

[16] Ogbulie, T. E., Ogbuilie J. N, and Njoku, H. O. (2007): comparative study on the shelf life statbility of palm wine from Elaeis guineensis and Raphiahookeriobtained from okigwe, Nigeria. African Journal of Biotechnology 6(7): 914-922.

[17] Gaffa T, Azoro C. (2005): Bacteriology of Biology, caterers and food technology. First published. Amana printing. Pp. 95-96, 104-105.

[18] Lyumugabe, F., Kamaliza, G., Bajyana, E., and Thonart P. H. (2010). Microbiological andphysico-chemical characteristic of Rwandese traditional beer "Ikigage, African Journal of Biotechnology, 9(27): 4241-4246.

[19] Navarrete-Bolaños, J.L. (2012). Improving traditional fermented beverages: how to evolve from spontaneous to directed fermentation: guidelines to design efficient directed fermentations based on spontaneous fermentations. Eng. Life Sci. 12, 410-418.doi:10.1002/elsc.201100128.

[20] Stringini,M., Comitini,F., Taccari,M., and Ciani,M. (2009). Yeast diversity during tapping and fermentation of palm wine from Cameroon. Food Microbiol. 26, 415-420.doi:10.1016/j.fm.2009.02.006.

[21] Elijah, A. I; Ojimelukwe, P.C.; Ekong, U. S.; Asamudo, N.U. (2010). Effect of Saco glottis gabonensis and Alstoniaboonei on the kinetics of Saccharomyces cerevisiae isolated from palm wine. African Journal of Biotechnology. 9(35): 5730-5734. http://www.academicjournals.org/AJB.

[22] Djeni, T. N., Karen H. Kouame, Francine D. M. Ake, Laurent S. T. Amoikon, Marcellin K. Dje and Kumara swamyJeyaram. Microbial Diversity and Metabolite Profiles of Palm Wine Produced From Three Different Palm Tree Species in côte d'ivoire. Scientific reports (2020) 10:1715 https://doi.org/10.1038/s41598-020-58587-2.

[23] Ganucci, D., Guerrini, S., Mangani, S., Vincenzini, M., and Granchi, L. (2018). Quantifying the effects of ethanol and temperature on the fitness advantage of predominant Saccharomyces cerevisiae strains occurring in spontaneous wine fermentations. Front. Microbiol. 9:1563. doi:10.3389/fmicb.2018.01563

[24] Johansen, P. G., Owusu-Kwarteng James, Parkouda Charles, S. WilfridPadonou and Lene Jespersen. Yeasts as Contributors to Spontaneously Fermented Food and Beverages Produced in Sub-Saharan Africa. Frontiers in Microbiology. August 2019, vol 10, Article 1789.

[25] Savadogo, A; Ouattara, C.A; Bassole, I.H; and Traore, S.A. (2006). Bacteriocin and lactic acid bacteria- A mini review. African journal of biotechnology 5(9): 678-683.

[26] J. M. Willey, L. Sherwood, C.J.Woolverton, L. M. Prescott: Prescott, Harley, and Klein's Microbiology. 7th ed New York: McGraw-Hill Higher Education, 2008. Ch 31, pp. 763.

Citation: E.J. Olotu et.al., (2020). "Therapeutic Potential of Palm Wine on Selected Diarrhoeagenic Bacteria”, International Journal of Clinical Chemistry and Laboratory Medicine (IJCCLM), 6(1), pp.17-24. DOI: http://dx.doi.org/10.20431/2455-7153.0601004

Copyright: (C) 2020 Authors, This is an open-access article distributed under the terms of the Creative Commons Attribution License, which permits unrestricted use, distribution, and reproduction in any medium, provided the original author and source are credited. 